\title{
Blue laser magnifying endoscopy in the diagnosis of chronic gastritis
}

\author{
YING ZHU ${ }^{1}$, FANG WANG ${ }^{1}$, YAN ZHOU ${ }^{2}$, GUI-LI XIA ${ }^{1}$, \\ LING DONG ${ }^{1}$, WEN-HUA HE ${ }^{1}$ and BING XIAO ${ }^{3}$

\begin{abstract}
${ }^{1}$ Department of Gastroenterology, Shenzhen Hospital of Southern Medical University, Shenzhen, Guangdong 518100;
${ }^{2}$ Department of Information Management Section, Bethune International Peace Hospital, Shijiazhuang, Hebei 050000;

${ }^{3}$ Department of Gastroenterology, Nanfang Hospital, Southern Medical University, Guangzhou, Guangdong 510515, P.R. China
\end{abstract}

Received August 14, 2018; Accepted June 6, 2019

DOI: $10.3892 /$ etm.2019.7811

\begin{abstract}
The current study aimed to evaluate the clinical value of using blue laser imaging combined with magnifying endoscopy in the diagnosis of chronic gastritis (CG). The groups used were as follows: The white light group (WLI, control group), linked color imaging group (LCI, observation group 1), blue laser imaging (BLI)-bright (brt) group (BLI-brt; observation group 2), BLI + magnified imaging (ME) group (observation group 3). WLI mode initially allowed the observation of mucosal suspicious lesions on the gastric mucosa. These lesions were photographed and the mode was changed to LCI, BLI-brt and BLI + ME. Different observational patterns were compared between modes to diagnose various grades of chronic gastritis. No significant differences were observed in the baseline information of enrolled patients. The LCI mode diagnosis rate was higher for Helicobacter pylori (HP) infection than in any other mode. LCI exhibited a high diagnostic rate for HP, BLI-brt exhibited a high diagnostic rate for atrophy and BLI/BLI + ME exhibited a high diagnostic rate for intestinal metaplasia and intraepithelial neoplasia. All modes exhibited higher diagnostic rates compared with the WLI mode. The pathological HP diagnosis rate (consistency) of HP infection was the greatest in the LCI group (endoscopic findings and pathological consistency). The BLI-BRT mode exhibited the highest pathological diagnosis rate for atrophic gastritis and the BLI/BLI + ME mode exhibited the highest diagnostic rate for intestinal metaplasia and low-grade intraepithelial neoplasia.
\end{abstract}

Correspondence to: $\mathrm{Dr}$ Bing Xiao, Department of Gastroenterology, Nanfang Hospital, Southern Medical University, 1838 North of Guangzhou Avenue, Guangzhou, Guangdong 510515, P.R. China

E-mail: fjxb@163.com

Key words: chronic gastritis, blue laser magnifying endoscopy

\section{Introduction}

Chronic gastritis (CG) is the chronic inflammation of the gastric mucosa that is induced by a variety of factors, but mainly by infection with Helicobacter pylori (HP) bacteria $(1,2)$. CG develops subsequent to the infiltration of lymphocytes and plasma cells in the gastric mucosa. Patients with CG may exhibit atrophy and intestinal metaplasia in the gastric mucosa in later stages of the condition $(3,4)$. The incidence of $\mathrm{CG}$ is high in the Chinese population, with $\sim 30 \%$ afflicted chronic gastritis. (5). Controversy exists as to the diagnosis and classification of CG, and these have resulted in varying classification methods using X-ray, immunology, endoscopy and histopathology analysis. Numerous classification schemes $(>10)$ exist regarding chronic gastritis worldwide $(6,7)$. The most well renowned of which is The New Sydney System Taxonomy (Updated Sydney System). However, due to the controversy over various diagnostic methods used, this system has yet to be implemented in China. The formulation of the earliest consensus opinions was undertaken in China in 1982. The consensus was updated in 2000, 2003 and 2006 and until November 2012, the national consensus conference on the diagnosis and treatment of CG was held in Shanghai. This conference provided regulations regarding the use of endoscopy in the diagnosis of this condition. An association has been previously identified between CG and gastric cancer $(8,9)$. A number of worldwide studies have demonstrated that the chronic inflammation of the gastric mucosa is closely associated with the occurrence of gastric cancer, and that gastric cancer is one of the most common malignancies in China $(10,11)$. The occurrence development model is as follows: (i) Normal gastric mucosa; (ii) chronic superficial gastritis; (iii) chronic atrophic gastritis; (iv) intestinal metaplasia; (v) dysplasia; and (vi) intestinal gastric cancer (12). The standard endoscopic diagnosis of chronic gastritis, the classification of endoscopy and the histological grade are essential in the discovery of early gastric cancer. Blue laser imaging with magnifying endoscopy provides endoscopic surgeons with a more accurate diagnosis of CG.

The blue laser imaging (BLI) technology was a novel treatment introduced globally in $2012(13,14)$. BLI replaces the traditional xenon lamp with a laser light source and performs white light and bright, narrow-band light observations (15). 
Through the use of lasers, new methods have been established for the diagnosis of various diseases using endoscopy. In recent years, narrow-band imaging (NBI) has been the most sophisticated gastroscopy method, which is used in clinical practice, in the endoscopic diagnosis of $\mathrm{CG}$ and early gastric cancer $(16,17)$. The high diagnostic value of NBI in the diagnosis of early gastrointestinal cancer has also been supported (18). However, due to the low brightness of NBI, wide gastric cavity and the limited mucosa visibility, various limitations of using this method in the diagnosis of gastric mucosal lesions exist. In BLI, the laser is the light source and the fusion images of the digestive tract mucosa are captured at wavelengths of 415 and $450 \mathrm{~nm}$ and provide increased penetration $(13,14)$. In addition to clear and high quality images produced by blue laser imaging, magnified observations can allow the assessment of deeper blood vessel structures in the mucosal membrane (15). This aids in the discovery of subtle structural changes in the mucosal surface and can help endoscopic doctors to accurately evaluate the lesions nature (19). In 2015, BLI endoscopy was introduced in various large and medium-sized urban hospitals in China (20). According to current data, compared with traditional endoscopy, BLI endoscopy diagnostic rate of the upper gastrointestinal tract and colorectal cancer was significantly increased, especially in younger patients $(21,22)$. The use of blue laser endoscopy has enabled doctors to make a more accurate diagnosis of observed lesions. Therefore, this imaging technique is clinically useful for the diagnosis of CG and for providing an accurate endoscopic classification and histological grade.

\section{Materials and methods}

Patients and grouping. The current study is a prospective study. Patients were selected if they had undergone blue laser magnifying endoscopy from August 2017 to July 2018 in the outpatient department at the Shenzhen Hospital of Southern Medical University (Shenzhen, China). Endoscopy and pathological biopsy were used to diagnose CG in 120 patients (60 male and 60 female), aged 26-79 years old. Four modes were used to observe all patients, which were divided into four groups. The groups were as follows: i) white light group (control group); ii) Linked color imaging (LCI) group (observation 1 group); iii) BLI-bright (BLI-brt) group (observation 2 group) and the iv) BLI+ magnified imaging (ME) group (observation 3 group). The diagnostic criteria used were based on the 'Consensus Opinion for Chronic Gastritis in China, 2012' of the Chinese Medical Association Gastroenterology Section (23). Endoscopic classification and histological grading were performed in 120 patients (23). All participants in the current study signed written informed consent. The current study is in full compliance with the ethical standards established by The Human Body Testing Committee of China.

Inclusion criteria. Patients meeting the diagnostic criteria were potentially eligible for the study if they met the following criteria: i) Aged between 26-79 years old; ii) being diagnosed as CG by western medicine procedures; iii) being diagnosed as $\mathrm{CG}$ according to syndrome differentiation in traditional Chinese medicine; and iv) voluntary submission of written informed consent prior to enrollment (24).
Exclusion criteria. The exclusion criteria were as follows: i) Functional dyspepsia (ruled out using medical history and endoscopy); ii) gastric and duodenal ulcers (ruled out using endoscopy); iii) gastric cancer (ruled out using endoscopic and pathological examination); iv) pancreatic and biliary diseases (ruled out using abdominal doppler ultrasonography) and partial computerized tomography and magnetic resonance were excluded if necessary; v) serum liver function was measured in all patients and 8 patients were excluded due to hepatitis; (vi) age <25 years, age $>79$ years, pregnant or lactating women; vii) cardiac, pulmonary and neuropsychiatric disorders, patients which cannot tolerate gastroscopy or patients who disagree with endoscopy; and viii) patients who had received anticoagulant drugs in preceding 2 weeks, or patients who exhibited coagulation dysfunction (25).

Inspection method. Using the EG-L590ZW electronic magnifying endoscope (FUJIFILM), combined with the LL-4450 laser light source (FUJIFILM Corporation) of the LASEREO blue laser endoscope system (FUJIFILM Corporation) and the VP-4450 HD image processing device (FUJIFILM Corporation), the gastric mucosa was initially observed using white light, and the mucosal suspicious lesions were photographed and observed using LCI. BLI-brt, BLI + ME images, biopsy under different observation methods, histopathological results and any necessary additional immunohistochemical examinations were performed to confirm the diagnosis.

Image analysis. The diagnosis concluded from endoscopy is subjective. Therefore, to avoid the selective bias in the current study, scientific and objective diagnoses were made. Two experienced endoscopists performed the endoscopies and two additional senior endoscopists randomly reviewed the results. Photographs, final endoscopic diagnosis and pathological results after review were used to measure the consistency between endoscopic diagnosis. When the endoscopic or pathological findings are controversial, an additional immunization group is used. The parameters of the immunization group were serum gastrin 17 (G-17), pepsinogen (PG I, PG II) and T cell subsets (CD4+T, CD8+T).

Pathological diagnosis. The gastric mucosa was observed using blue light and lesions were examined using four observational modes: The white light model (WLI); LCI; BLI-BRT; and the BLI/BLI + ME. The suspicious lesion sites were observed using these four observational modes aforementioned and any pathological signs: i) Helicobacter pylori infection; ii) chronic inflammatory response (mononuclear cell infiltration) and activity (neutrophil infiltration); iii) atrophy (decrease of inherent glands); and iv) and intestinal metaplasia were recorded. Comparative studies were subsequently performed. Images were first captured at typical locations of observed lesions, the samples of diseased tissues were then taken and stained using heterochromic reaction by toluidine blue, which is followed by pathological analysis. Comparative analysis of the diagnostic accuracy of the three examination methods (LCI, BLI-BRT and BLI/BLI + MI) was performed, and four methods (WLI, LCI, BLI-BRT and BLI/BLI + MI) were used for the endoscopic classification of chronic gastritis (based on the CG classification in 2012) into superficial gastritis, erosive gastritis, 
hemorrhagic gastritis and atrophic gastritis. Histological changes were also used in the disease classification: Chronic inflammatory response (+), active inflammation (++), atrophy $(+++)$ and intestinal metaplasia (++++) based on the degree of pathological changes $(26,27)$. Helicobacter pylori infection was considered as +/++ according to the literature (28).

Diagnosis. Chronic gastritis is currently considered to be indicated by a mucositis change, which can be observed by the naked eye or using special imaging methods under endoscopy. The final diagnosis should be concluded in conjunction with a pathological examination.

Statistical analysis. Data are presented as the mean \pm standard deviation. Statistical analysis was performed using SPSS version 13.0 (SPSS, Inc.). One-way ANOVA was used to detect the differences between groups of various treatments after establishing the distribution of the data and the equivalency of variances using SPSS version 13.0. The Student-Newman-Keuls test was used as the post-hoc test. $\mathrm{P}<0.05$ was considered to indicate a statistically significant difference.

\section{Results}

Patient characteristics. The same mode was used to observe the same 120 patients. Combined with pathological biopsy results, the diagnostic rate of blue laser magnifying endoscopy was evaluated and compared with that of normal white light imaging. The baseline information of the patient, including sex, age, severity of the disease, living environment and habits among the enrolled patients is shown in Tables I-VII. However, no significant differences were observed $(\mathrm{P}>0.05)$.

Images of the LCI pattern exhibited by HP infection. A representative image of a patient of each group in this study was selected for observation (Fig. 1). The current study revealed that the diagnosis of HP infection using LCI was higher compared with the other modes. The red-white boundary and abnormal redness can be observed under endoscopy. The abnormal redness is divided into four appearances: Stove-like redness, flat erosion, uplift erosion and diffuse redness.

Low-level intraepithelial neoplasia. A representative image of a patient was selected in this study for observation (Fig. 2). The WLI and LCI model observation revealed inflammation. Lesions were rough compared with the surrounding mucosa. The BLI-brt model observed the lesions in comparison with the background and revealed a brown color, which determined the extent of lesions. The BLI + ME observation model showed no obvious demarcation line (DL), regular micro surface (MS) patterns, micro vascular (MV) patterns, distortions or irregularities or low-grade intraepithelial neoplasia (LGIN).

Chronic atrophic gastritis observation modes. A representative image of a patient was selected in this study for observation (Fig. 3). The WLI, LCI, BLI-brt observation model showed that the lesions were slightly rough compared with the surrounding mucosa. The mucosa color appeared 
Table II. Distribution of gastritis grading in each group of patients.

\begin{tabular}{lcccc}
\hline & \multicolumn{3}{c}{ Pathological typing (n) } \\
\cline { 2 - 4 } Group & None & Mild & Moderate & Severe \\
\hline White light mode & 0 & 116 & 4 & 0 \\
Linked color imaging & 0 & 78 & 38 & 4 \\
Blue laser imaging-bright & 0 & 113 & 5 & 0 \\
Blue laser imaging/ & 0 & 116 & 4 & 0 \\
Blue laser imaging + magnified imaging & & & & \\
\hline
\end{tabular}

Table III. Distribution of gastritis activity classification in each group of patients.

\begin{tabular}{lrrrr}
\hline & \multicolumn{3}{c}{ Pathological typing (n) } \\
\cline { 2 - 4 } Group & None & Mild & Moderate & Severe \\
\hline White light mode & 116 & 0 & 113 & 116 \\
Linked color imaging & 78 & 35 & 3 & 0 \\
Blue laser imaging-bright & 113 & 4 & 2 & 2 \\
Blue laser imaging/ & 116 & 3 & 2 & 2 \\
Blue laser imaging + magnified imaging & & & & \\
\hline
\end{tabular}

Table IV. Distribution of HP classification in each group of patients.

\begin{tabular}{lcccc}
\hline & \multicolumn{3}{c}{ Pathological typing (n) } \\
\cline { 2 - 4 } Group & None & Mild & Moderate & Severe \\
\hline White light mode & 116 & 0 & 2 & 3 \\
Linked color imaging & 78 & 35 & 4 & 3 \\
Blue laser imaging-bright & 113 & 3 & 2 & 2 \\
Blue laser imaging/ & 116 & 0 & & 2 \\
Blue laser imaging + magnified imaging & & & 2 \\
\hline
\end{tabular}

Table V. Distribution of intestinal differentiation grading in each group of patients.

\begin{tabular}{lcccc}
\hline & \multicolumn{3}{c}{ Pathological typing (n) } \\
\cline { 2 - 4 } Group & None & Mild & Moderate & Severe \\
\hline White light mode & 116 & 0 & 2 & 2 \\
Linked color imaging & 114 & 1 & 3 & 2 \\
Blue laser imaging-bright & 109 & 3 & 6 & 2 \\
Blue laser imaging/ & 65 & 44 & & 3 \\
Blue laser imaging + magnified imaging & & & \\
\hline
\end{tabular}

to be different in the different modes. However, in all cases, an obvious color difference was observed compared with the surrounding normal mucosa. Under the BLI + ME observation mode, regular MS pattern, gland shortening and a spiral blood vessel were observed between the fossa.
Various observational modes of intestinal metaplasia (GIM). A representative image of a patient was selected in this study for observation (Fig. 4). LCI mode observation revealed rough lesions, uneven color, BLI + ME weakly magnified the visible DL, a strong amplifying gland typical finger shape and regular MV pattern. 
Table VI. Distribution of stomach atrophy grading in each group of patients.

Pathological typing (n)

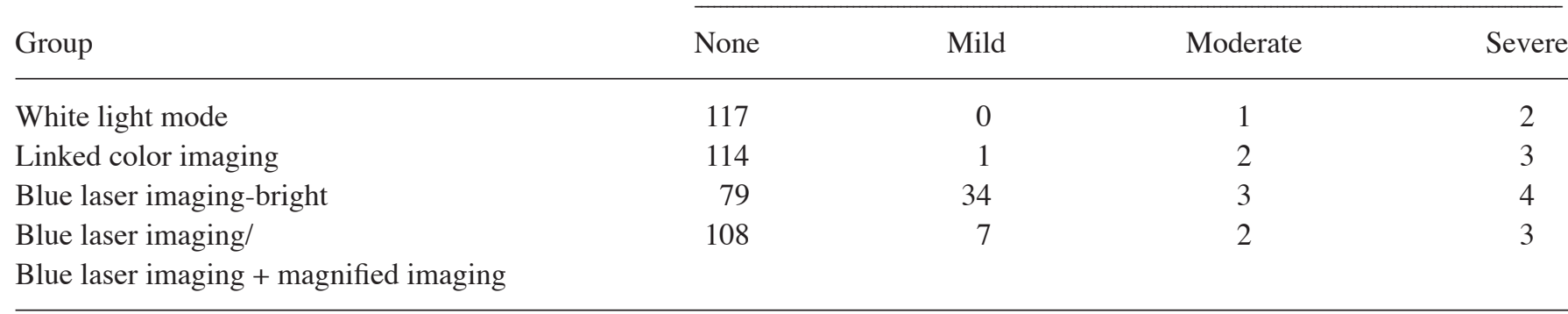

Table VII. Distribution of epithelial sarcoma grading in each group of patients.

\begin{tabular}{lcr}
\hline & \multicolumn{2}{c}{ Pathological typing (n) } \\
\cline { 2 - 3 } Group & None & Mild \\
\hline White light mode & 0 & 1 \\
Linked color imaging & 0 & 3 \\
Blue laser imaging-bright & 0 & 11 \\
Blue laser imaging/ & 0 & 31 \\
Blue laser imaging + & & \\
magnified imaging & &
\end{tabular}

\section{Discussion}

In the present study, BLI technology was used to aid in the accurate diagnosis and classification of chronic gastritis via endoscopy, standardize histological grading, establish contrast imaging with traditional white light, in combination with histopathology. The current study assessed the clinical value of this technology. BLI remains to be a novel technology, therefore, the number of cases of its use are insufficient. Studies assessing a large sample size of multi-center clinical studies are required to further support the use of this technique clinically.

An LCI pattern was observed in patients exhibiting HP infection. In this mode, the sinusoidal junction demonstrated a clear red-white boundary, and the targeted biopsy results suggested the presence of a HP infection. Additionally, the mucosa at the gastric fundus was diffuse and red. In the WLI mode, mucosal roughness was observed in the central stomach angle, which suggested the presence of inflammation. Irregular redness was observed in the central stomach angle, and the center was slightly depressed, which may have been caused by the inflammatory reaction. In the BLI-brt mode, the middle part of the stomach was brown with no obvious observed boundaries. In the BLI + ME mode, no DL, regular MS pattern, MV irregularity, distortion, targeted pathology or low-grade intraepithelial neoplasia.

In the WLI mode, the gastric mucosa was not smooth. In the LCI mode, the gastric mucosa was not smooth, uneven, with some of the mucous membranes exhibiting an orange

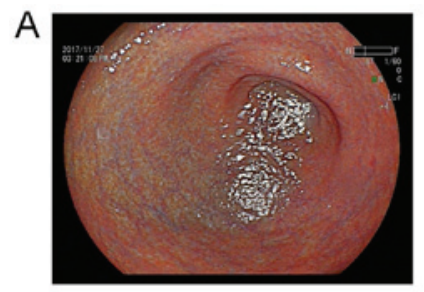

sinudoidal junction

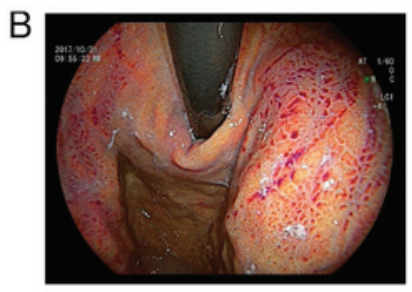

mucosa at the gastric fundus
Figure 1. Typical image of a Helicobacter pylori infection, observed using linked color imaging. Images using the linked color imaging mode of (A) the sinusoidal junction and (B) the mucosa at the gastric fundus. Both images were taken from the same patient.

color. The BLI-brt mode revealed that the lesions in the stomach horn were rougher than the surrounding mucosa and indicated a brown color. Under the BLI magnification mode, the MS pattern of the gastrointestinal angle was observed, the gland was shortened and the pathology showed atrophy.

From the various observational modes of visualizing GIM in the present study, it has been revealed that the mucosa of the gastric antrum anterior wall was white in the WLI mode. In the LCI mode, the mucosa of the antral antrum anterior wall was swollen and rough. DL was observed in the BLI weak amplification mode and the lesions center exhibited a brown color. BLI was clear in the strong amplification mode, the glands were observed to be finger-like, the MV pattern was regular and the targeted biopsy indicated intestinal metaplasia.

The diagnosis of chronic gastritis is commonly based on pathological diagnosis (29). The coincidence rate of endoscopic and pathological diagnosis of atrophic gastritis is low. The reason for this is the subjective analysis by endoscopic surgeons, the selection of endoscopic examination and sampling site inaccuracy (30). Blue laser magnifying endoscopy (BLI-bright/BLI + amplification) allows a clear observation of the fine morphology of blood vessels and glands and, combined with the clinical classification, can help doctors to accurately determine the nature of lesions, reduce biopsy sites, increase the positive rate of biopsy, reduce the rate of missed diagnosis and increase the detection of early stage gastric cancer.

The use of blue laser technology can increase the diagnostic rate of chronic gastritis under endoscopy, and can also increase the diagnostic rate of early gastric cancer, 

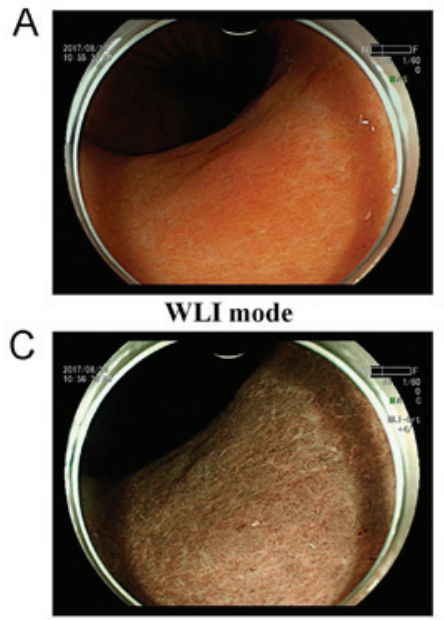

BLI-brt mode

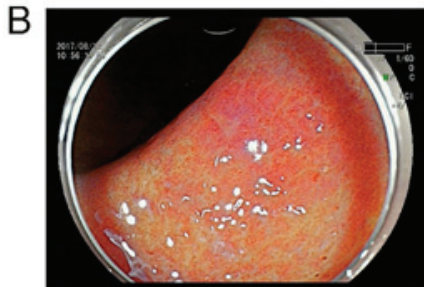

LCI mode

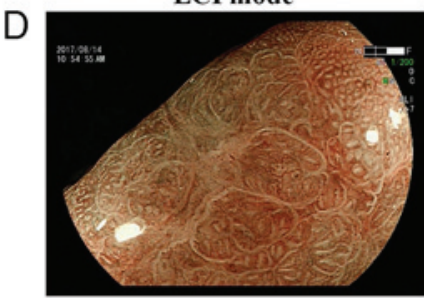

BLI+ME mode

Figure 2. Low-level intraepithelial neoplasia. Captured images of the (A) white light imaging mode, (B) linked color imaging mode, (C) blue laser imaging-bright mode and (D) blue laser imaging + magnified imaging mode used on the central stomach angle. All images were taken from the same patient.

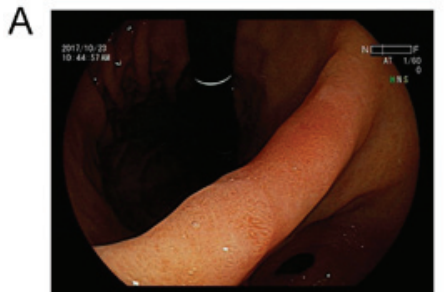

WLI mode

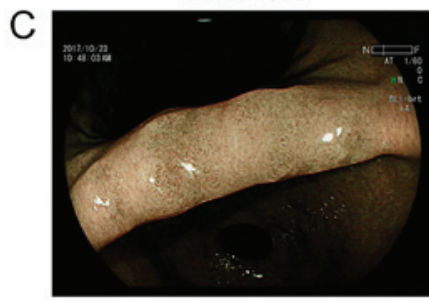

BLI-brt mode

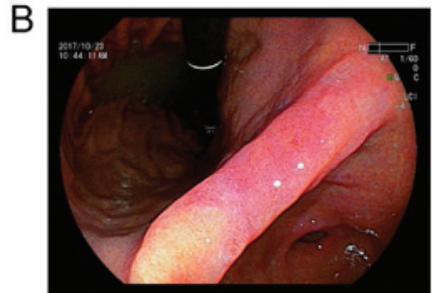

LCI mode

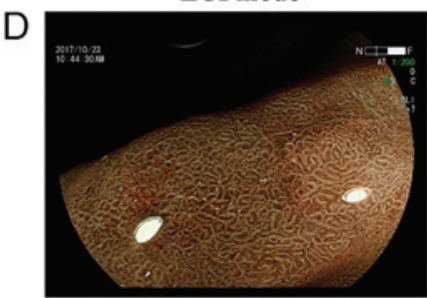

BLI+ME mode

Figure 3. Chronic atrophic gastritis observation modes. Images captured of the (A) white light imaging mode, (B) linked color imaging modeused to observe the gastric mucosa. Images captured of the (C) blue laser imaging-bright mode observations of a lesion of the stomach horn. Images captured of the (D) blue laser imaging magnification mode observations of the micro surface regular pattern of the gastrointestinal angle.All images were taken from the same patient.
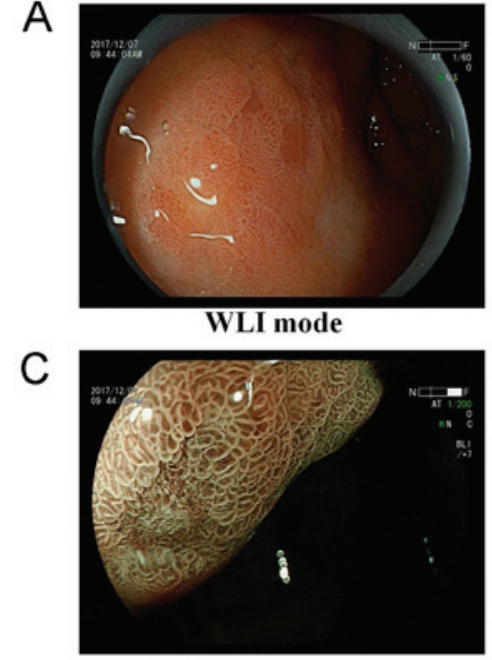

BLI-brt mode

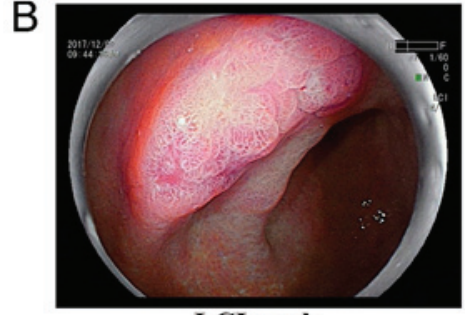

LCI mode

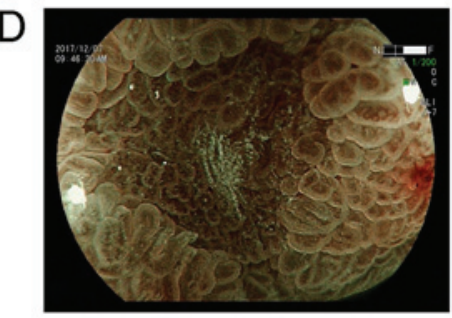

BLI+ME mode

Figure 4. Various observational modes of intestinal metaplasia. Captured images of (A) the white light imaging mode, (B) linked color imaging mode, (C) blue laser imaging weak amplification mode and (D) blue laser imaging strong amplification mode observations of the gastric antrum anterior wall. All images were taken from the same patient. 
HP infection, atrophic gastritis, intestinal metaplasia and intraepithelial neoplasia. The blue laser imaging technology provides clear, three-dimensional imaging compared with the traditional white light endoscopy technology. Different grades of chronic gastritis can be observed via endoscopy examinations. The current study has demonstrated that blue laser imaging can improve the screening ability of gastric mucosal diseases.

In several modes, the pathological diagnosis rate (consistency) of HP infection is the best in all pathological types of LCI group (endoscopic findings and pathological consistency). The BLI-brt mode exhibited the highest pathological diagnosis rate for atrophic gastritis. BLI/BLI + ME exhibited the highest diagnostic rate for intestinal metaplasia and low-grade intraepithelial neoplasia.

In conclusion, the present study showed that the use of blue laser endoscopy enabled doctors to diagnose observed lesions more accurately during the diagnosis of chronic gastritis. Therefore, blue laser endoscopy technology can be used in the clinical diagnosis of CG and provide accurate endoscopy classification and histological grading.

\section{Acknowledgements}

Not applicable.

\section{Funding}

The present study was supported by The Basic Research Subject of Science and Technology plan in Bao'an District Bao'an District (grant no. 2017JD167).

\section{Availability of data and materials}

The datasets used and/or analyzed during the current study are available from the corresponding author on reasonable request.

\section{Authors' contributions}

YiZ was responsible for guaranteeing integrity of the entire study, study concepts and design, definition of intellectual content, literature research, clinical studies, experimental studies, data acquisition, manuscript preparation and editing and review. FW was responsible for guaranteeing integrity of the entire study, study concepts, definition of intellectual content, experimental studies and data acquisition. YaZ was responsible for the experimental studies. GLX was responsible for the experimental studies and manuscript editing. LD was responsible for the experimental studies, manuscript editing and review. WHH was responsible for the literature research, clinical studies, experimental studies, data acquisition and analysis, statistical analysis, manuscript preparation and editing and review. BX was responsible for the entire study, study concepts and design. All authors read and approved the final manuscript.

\section{Ethics approval and consent to participate}

The study was approved by the Shenzhen Hospital of Southern Medical University (Shenzhen, China). Written informed consent was obtained.

\section{Patient consent to participate}

The current study is in full compliance with the ethical standards established by The Human Body Testing Committee of China.

\section{Competing interests}

The authors declare that they have no competing interests.

\section{References}

1. Colakoğlu EC,, Börkü K, Haydardedeoğlu AE, Alihosseini H, Şenel OO, Yumuşak N, Özen D, Baş B and Uğurlu L: Correlation between endoscopic and histopathological findings in dogs with chronic gastritis. J Vet Res 61: 351-355, 2017.

2. Liu Y, Gong H, Liu J and Zhang H: Acupoint selection pattern of chronic atrophic gastritis based on data mining methods of latent structure model and frequency item set. Zhongguo Zhen Jiu (Chinese) 38: 667-671, 2018.

3. Cherdantseva LA, Potapova OV, Sharkova TV, Belyaeva YY and Shkurupiy VA: Association of Helicobacter pylori and iNOS production by macrophages and lymphocytes in the gastric mucosa in chronic gastritis. J Immunol Res 2014: 762514, 2014.

4. Sonnenberg A and Genta RM: Prevalence of benign gastric polyps in a large pathology database. Dig Liver Dis 47: 164-169, 2015.

5. Jiang JX, Liu Q, Zhao B, Zhang HH, Sang HM, Djaleel SM, Zhang GX and Xu SF: Risk factors for intestinal metaplasia in a southeastern Chinese population: An analysis of 28,745 cases. J Cancer Res Clin Oncol 143: 409-418, 2017.

6. Tsimmerman YS and Zakharova YA: Kyoto consensus-The new etiological classification of chronic gastritis and its discussion. Klin Med (Mosk) (Russian) 95: 181-188, 2017.

7. Tsimmerman YS and Zakharova YA: Topical problems of chronic gastritis. Klin Med (Mosk) (Russian) 95: 8-14, 2017.

8. Yoon $\mathrm{H}$ and Kim N: Diagnosis and management of high risk group for gastric cancer. Gut Liver 9: 5-17, 2015.

9. Pasechnikov V, Chukov S, Fedorov E, Kikuste 1 and Leja M: Gastric cancer: Prevention, screening and early diagnosis. World J Gastroenterol 20: 13842-13862, 2014.

10. Rudnicka K, Backert S and Chmiela M: Genetic polymorphisms in inflammatory and other regulators in gastric cancer: Risks and clinical consequences. Curr Top Microbiol Immunol 421: 53-76, 2019.

11. Strong VE, Wu AW, Selby LV, Gonen M, Hsu M, Song KY, Park CH, Coit DG, Ji JF and Brennan MF: Differences in gastric cancer survival between the U.S and China. J Surg Oncol 112: 31-37, 2015.

12. Karimi P, Islami F, Anandasabapathy S, Freedman ND and Kamangar F: Gastric cancer: Descriptive epidemiology, risk factors, screening, and prevention. Cancer Epidemiol Biomarkers Prev 23: 700-713, 2014.

13. Yoshida N, Dohi O, Inoue K, Yasuda R, Murakami T, Hirose R, Inoue K, Naito Y, Inada Y, Ogiso K, et al: Blue laser imaging, blue light imaging, and linked color imaging for the detection and characterization of colorectal tumors. Gut Liver 13: 140-148, 2019.

14. Nakashima H, Kawahira H, Kawachi H and Sakaki N: Artificial intelligence diagnosis of Helicobacter pylori infection using blue laser imaging-bright and linked color imaging: A single-center prospective study. Ann Gastroenterol 31: 462-468, 2018.

15. Togashi K, Nemoto D, Utano K, Isohata N, Kumamoto K, Endo S and Lefor AK: Blue laser imaging endoscopy system for the early detection and characterization of colorectal lesions: A guide for the endoscopist. Therap Adv Gastroenterol 9: 50-56, 2016.

16. Horiguchi N, Tahara T, Kawamura T, Okubo M, Tahara S, Nagasaka M, Nakagawa Y, Shibata T and Ohmiya N: A comparative study of white light endoscopy, chromoendoscopy and magnifying endoscopy with narrow band imaging in the diagnosis of early gastric cancer after Helicobacter pylori eradication. J Gastrointestin Liver Dis 26: 357-362, 2017.

17. Osawa $\mathrm{H}$ and Yamamoto $\mathrm{H}$ : Present and future status of flexible spectral imaging color enhancement and blue laser imaging technology. Dig Endosc Suppl 1: 105-115, 2014.

18. Zhou F, Wu L, Huang M, Jin Q, Qin Y and Chen J: The accuracy of magnifying narrow band imaging (ME-NBI) in distinguishing between cancerous and noncancerous gastric lesions: A meta-analysis. Medicine (Baltimore) 97: e9780, 2018. 
19. Nagahama T, Yao K, Uedo N, Doyama H, Ueo T, Uchita K, Ishikawa H, Kanesaka T, Takeda Y, Wada K, et al: Delineation of the extent of early gastric cancer by magnifying narrow-band imaging and chromoendoscopy: A multicenter randomized controlled trial. Endoscopy 50: 566-576, 2018.

20. Diao W, Huang X, Shen L and Zeng Z: Diagnostic ability of blue laser imaging combined with magnifying endoscopy for early esophageal cancer. Dig Liver Dis 50: 1035-1040, 2018.

21. Cao HL, Dong WX, Xu MQ, Zhang YJ, Wang SN, Piao MY, Cao XC and Wang BM: Clinical features of upper gastrointestinal serrated lesions: An endoscopy database analysis of 98746 patients. World J Gastroenterol 22: 10038-10044, 2016.

22. Moehler M, Voigt J, Kastor M, Heil M, Sengespeick C, Biesterfeld S, Dippold W, Kanzler S and Galle PR: Endoscopic ultrasonography-guided fine-needle aspiration (EUS-FNA) as primary diagnostic tool for unclear lesions in the upper gastrointestinal tract. Dtsch Med Wochenschr (German) 136: 303-308, 2011.

23. Society of Digestive Diseases, Chinese Medical Association, Fang Jingyuan, Liu Wenzhong, et al: China Consensus on Chronic Gastritis (Shanghai, 2012). Chinese Journal of Digestion 33: 5-16, 2013.

24. Tian G, Wu C, Li J, Liang B, Zhang F, Fan X, Li Z, Wang Y, Li Z, Liu D, et al: Network pharmacology based investigation into the effect and mechanism of Modified Sijunzi Decoction against the subtypes of chronic atrophic gastritis. Pharmacol Res 144: $158-166,2019$.

25. Negovan A, Iancu M, Moldovan V, Sàrkàny K, Bataga S, Mocan S, Tilea I and Banescu C: The contribution of clinical and pathological predisposing factors to severe gastro-duodenal lesions in patients with long-term low-dose aspirin and proton pump inhibitor therapy. Eur J Intern Med 44: 62-66, 2017.
26. Fukuda H, Miura Y, Hayashi Y, Takezawa T, Ino Y, Okada M, Osawa H, Lefor AK and Yamamoto H: Linked color imaging technology facilitates early detection of flat gastric cancers. Clin J Gastroenterol 8: 385-389, 2015.

27. Guo XY, Dong L, Qin B, Jiang J and Shi AM: Decreased expression of gastrokine 1 in gastric mucosa of gastric cancer patients. World J Gastroenterol 20: 16702-16706, 2014.

28. Watari J, Chen N, Amenta PS, Fukui H, Oshima T, Tomita T, Miwa H, Lim KJ and Das KM: Helicobacter pylori associated chronic gastritis, clinical syndromes, precancerous lesions, and pathogenesis of gastric cancer development. World J Gastroenterol 20: 5461-5473, 2014.

29. Sipponen P and Maaroos HI: Chronic gastritis. Scand J Gastroenterol 50: 657-667, 2015

30. Kosztyła-Hojna B, Berger G and Zdrojkowski M: Phonosurgical methods of treatment in unilateral vocal folds paralysis. Pol Merkur Lekarski (Polish) 42: 173-177, 2017. 